\title{
TAGUNG
}

\section{Für ein solidarisches und solides Europa}

\author{
Frédéric Krumbein*
}

Nach der Begrüßung durch Michèle Knodt sagte Peter-Christian Müller-Graff, die Tagung inhaltlich einleitend, dass die Krisenstaaten der Europäischen Union sich aktuell Beistand unter dem Stichwort der Solidarität wünschten und dass dieser Beistand nicht verweigert werde. Die Hilfe für krisengeplagte Eurostaaten habe heftige Diskussionen in den Geber- und Nehmerstaaten ausgelöst und es habe in Deutschland noch nie eine so intensive Unterstützung für Verfassungsklagen gegeben wie für diejenigen, die sich gegen die Krisenhilfen für überschuldete Eurostaaten wandten. Deutschland sei unvermittelt in die Rolle des primären Retters des Euros hineingeraten.

\section{Ein stärkeres Kerneuropa durch eine} Verkleinerung der EU?

Andreas Maurer sprach über Desintegration als mögliche Chance der Integration. Zunächst definierte er den Integrationsbegriff in Europa: Merkmale der Integration bestünden in der Schaffung eines gemeinsamen Marktes und eines gemeinsamen Regelsystems, in der $\mathrm{Zu}$ sammenarbeit zwischen den verschiedenen Ebenen im EU-Mehrebenensystem, der Etablierung von gemeinsamen Institutionen sowie der Zieloffenheit der europäischen Einigung. Symmetrie und Asymmetrie der Integrationsprozesse, wie eine existierende Währungsunion bei gleichzeitig unvollendeten anderen Bereichen der europäischen Integration, und ein Integrationsprozess in unregelmäßigen Schritten stellten weitere Merkmale dar.

Die wachsenden Klüfte zwischen Zentrum und Peripherie in der Europäischen Union sowie

\section{Projekt Europa - Zwischen Solidarität und Desintegration}

11. Dreiländertagung von ECSA Austria, ECSA Suisse und dem Arbeitskreis Europäische Integration e.V. und Jahreskolloquium des Arbeitskreises Europäische Integration e.V.

Mit finanzieller Unterstützung der Europäischen Kommission und der Carlo und Karin Giersch-Stiftung

Darmstadt, 15./16. November 2012

\section{Begrüßung und Einführung}

Prof. Michèle KNODT, Technische Universität Darmstadt

Prof. Peter-Christian MÜLLER-GRAFF, Arbeitskreis Europäische Integration und Universität Heidelberg

\section{Grundfragen des Projekts Europa}

Moderation: Prof. Peter-Christian MÜLLERGRAFF, Arbeitskreis Europäische Integration und Universität Heidelberg

Desintegration als Chance der Integration?

Dr. Andreas MAURER, Stiftung Wissenschaft und Politik, Brüssel

Solidarität als Rechtsprinzip der Europäischen Union

Dr. Marcus KLAMERT, Wirtschaftsuniversität Wien

Einzelfragen von Solidarität und Desintegration (1)

Moderation: Prof. Richard SENTI, Eidgenössische Technische Hochschule Zürich

Solidarität vs. ,, moral hazard“ in der Staatsschuldenkrise

Dr. habil. Christian DEUBNER, IES Abroad, Ber$\operatorname{lin}$

* Dr. Frédéric Krumbein, Geschäftsführer des Arbeitskreises Europäische Integration, Berlin. 
die ökonomische Abkopplung einiger Regionen würden im Gegensatz dazu oft zu den Phänomenen der Desintegration gezählt. Der britischen Haltung liege ein anderes Verhältnis zur Europäischen Union zugrunde. Dabei betrachteten die britischen Befürworter einer anderen Rolle Großbritanniens in der Europäischen Union ihre Haltung eher als Debattenbeiträge (,voice“), die für ein anderes Ziel der europäischen Integration optierten, und nicht als Absichtserklärungen für einen Austritt und damit als Desintegration. Die Nichteinhaltung von EU-Normen, wie im Falle von Teilen der EU-Grundrechtecharta durch Ungarn, oder von EU-Regeln durch einzelne Mitgliedstaaten, wie im Fall des Stabilitäts- und Wachstumspakts, seien ebenfalls Formen von Desintegration. Wissenschaftlich erfasst sei der Begriff der Desintegration aber bislang kaum.

„Exit“ tauche als eine Handlungsoption, gerade in Bezug auf Großbritannien, immer wieder auf. „Voice“ stelle eine andere Handlungsoption dar, beispielsweise durch die Aushandlung von Opt-Outs, das heißt die Nichtbeteiligung an einzelnen vergemeinschafteten Politikfeldern. Bei Opt-Outs werde das Gesamtsystem nicht infrage gestellt, aber in einzelnen Politikbereichen erfolge keine Integration.

Ein zweiter Bereich sei die differenzierte Integration, bei der einige Staaten voranschritten, aber im Prinzip alle Staaten Ziel und Grundsätze des Integrationsschrittes anerkennen würden, wie zum Beispiel beim Schengenabkommen über den Verzicht auf Grenzkontrollen an den europäischen Binnengrenzen.

Eine dritte Möglichkeit bestehe darin, dass einzelne Staaten eine vertiefte Integration anstrebten und dabei nicht abhängig von Dritten, beispielsweise weniger integrationswilligen Staaten, sein wollten. Der Fiskalpakt sei ein Beispiel für diese dritte Möglichkeit. Er stehe für die "Sezession des Zentrums“ aus dem Gesamtgefüge der europäischen Integration und nicht umgekehrt für den Austritt der Periphe-
Die rechtliche Neuordnung der europäischen Wirtschafts- und Währungsunion

Prof. Peter-Christian MÜLLER-GRAFF, Arbeitskreis Europäische Integration und Universität Heidelberg

Einzelfragen von Solidarität und Desintegration (2)

Moderation: Prof. Fritz BREUSS, ECSA Austria und Wirtschaftsuniversität Wien

Ansätze für eine theoretische Konzeptionalisierung von Desintegration

Dr. Annegret EPPLER, Universität Tübingen, und Dr. Henrik SCHELLER, Universität Potsdam

Solidarität in der Energiepolitik

Nadine PIEFER, Technische Universität Darmstadt

Einzelfragen von Solidarität und Desintegration (3)

Moderation: Dr. Friedrich HEINEMANN, Arbeitskreis Europäische Integration und Zentrum für Europäische Wirtschaftsforschung, Mannheim

ESM und Fiskalpakt - Fortentwicklung der EU als Staatenverbund?

Dr. Franz CROMME, Staatssekretär a.D., Delmenhorst

rie. Frankreich und Deutschland wollten im Falle des Fiskalpakts keine Rücksichten auf die Interessen von Großbritannien und Tschechien nehmen, die nicht mit der deutsch-französischen Vorgehensweise einverstanden waren. Das einzige Argument für die Auslagerung des Fiskalpakts aus dem europäischen Primärrecht sei die Möglichkeit gewesen, den Pakt durch schnellere Verhandlungs- und Ratifikationsprozesse umzusetzen.

Desintegration könne vor allem als Chance für eine integrative Verstärkung eines Kerns der Union gesehen werden. Diese hätte aber den Ausschluss einzelner Staaten vom Integrationsprozess zum Preis. Diese Gruppe wünsche sich ein höheres Niveau der Integration, entsprechend der eigenen Definition, und nähme dabei keine Rücksicht auf die Interessen von Staaten, die möglicherweise eine andere Definition von Integration hätten. 
In der Europäischen Union bestehe gegenwärtig weder Einigung über die ,finalité " noch über einen gemeinsamen Prozess der Integration. Der Wille, für beides eine klare allgemein geteilte Definition zu finden, sei teilweise gering ausgeprägt, zum Beispiel bei Deutschland und Frankreich in Bezug auf die Berücksichtigung der Wünsche Großbritanniens.

Es folgte eine Diskussion über die Ziele der Europäischen Union. Müller-Graff wies daraufhin, dass die Förderung von Frieden und Wohlstand der Völker gemäß Art. 3 des Vertrags über die Europäische Union (EUV) ein Unionsziel darstellt. Maurer sah diese eher als abstrakte Ziele und die europäische Integration als einen kontinuierlichen Prozess ohne Ziel. Dieser immerwährende Prozess sei ein konstitutives Element der Union. Auf ein konkretes Ziel könnten sich die EU-Staaten nicht einigen.

Mathias Jopp bezweifelte, dass eine intergouvernementale Zusammenarbeit der Kernstaaten zu einer stärkeren Vertiefung der Union führen könne. So trete der deutsch-französische Gegensatz in vielen Politikfeldern offener zutage als im größeren Kreis aller 27 Mitgliedstaaten.

Wolfgang Kerber bemerkte, dass ein Kennzeichen der Währungsunion darin bestehe, dass viele Staaten die in der gegenwärtigen Krise notwendigen Integrationsschritte nicht wollten. Deutschland habe keine Haftungsverpflichtung gewollt und die südeuropäischen Staaten zeigten kein Interesse an den jetzt notwendigen Strukturreformen ihrer Volkswirtschaften. Die weitverbreitete Unzufriedenheit in den Bevölkerungen vieler EU-Staaten resultiere aus diesen unfreiwilligen Integrationsschritten.

Solidarität im EU-Recht: Häufig erwähnt, aber selten mit rechtsverbindlichen Folgen

Marcus Klamert referierte anschließend über Solidarität als Rechtsprinzip der Europäischen Union. Solidarität als umgangssprachlicher Begriff bezeichne die gegenseitige Hilfe. So- lidarität im EU-Recht stelle ein Verfassungsprinzip dar und solle unter anderem die Einheit des Unionsrechts befördern und leite sich aus Verfassungstraditionen, der Europäischen Menschenrechtskonvention oder anderen grundlegenden Rechtsdokumenten.

Bei einem Vergleich der beiden Rechtsprinzipien Loyalität und Solidarität zeigten sich Unterschiede. Loyalität kennzeichne sein systemischer Charakter, das heißt es finde sich im EU-Recht seine Vollstreckbarkeit, die Rechtsfortbildung durch dieses Prinzip und eine überwiegend vertikale Anwendung im europäischen Mehrebenensystem. Pacta sunt servanda stelle ein klassisches Beispiel des Rechtsprinzips der Loyalität im Völkerrecht dar.

Solidarität habe ebenfalls einen systemischen Charakter. Aber darüber hinaus bestünden Unterschiede zum Loyalitätsprinzip. Es stelle sich die Frage der Vollstreckbarkeit des Solidaritätsprinzips, die eher zu verneinen wäre. Solidarität könne überwiegend als ein politisches Prinzip betrachtet werden und es sei eher horizontal als vertikal ausgerichtet. Im Völkerrecht werde Solidarität vor allem im Bereich des Katastrophenschutzes oder beim Prinzip der Meistbegünstigung im Rahmen der Welthandelsorganisation erwähnt. Gemeinsamkeiten beider Prinzipien zeigten sich im kooperativen, wertebasierten und existentiellen Charakter.

In den Verträgen werde Solidarität in der Präambel und in Art. 3 EUV erwähnt: Genannt seien die Solidarität zwischen den Völkern, den Generationen und den Mitgliedstaaten. Im Bereich der Wirtschaftspolitik erwähne Art. 122 des Vertrags über die Arbeitsweise der Europäischen Union (AEUV) die Solidarität: „Der Rat kann auf Vorschlag der Kommission unbeschadet der sonstigen in den Verträgen vorgesehenen Verfahren im Geiste der Solidarität zwischen den Mitgliedstaaten über die der Wirtschaftslage angemessenen Maßnahmen beschließen“.

In der Asylpolitik fänden sich in den Art. 67 und 80 AEUV Hinweise auf das Solidaritäts- 
prinzip, die eine gerechte Aufteilung der Verantwortlichkeiten für Asylsuchende zwischen den Mitgliedstaaten vorsehe. In der Energiepolitik werde Solidarität ebenfalls erwähnt. Die bekannteste Erwähnung der Solidarität finde sich in Art. 222 EUV, der sogenannten Solidaritätsklausel, die beispielsweise die gegenseitige Unterstützung der Mitgliedstaaten bei Naturkatastrophen beinhalte. In der EUGrundrechtecharta werde Solidarität häufig erwähnt, beispielsweise bei den Arbeitnehmerrechten, der sozialen Sicherheit oder der Gesundheitsversorgung.

Im Rahmen der Gemeinsamen Außen- und Sicherheitspolitik (GASP) finde sich das Prinzip der Solidarität ebenfalls in den EU-Verträgen, beispielsweise die sogenannte ,Solidaritätsklausel“ des Art. 24 Abs. 3 EUV: „Die Mitgliedstaaten unterstützen die Außen- und Sicherheitspolitik der Union aktiv und vorbehaltlos im Geiste der Loyalität und der gegenseitigen Solidarität und achten das Handeln der Union in diesem Bereich. Die Mitgliedstaaten arbeiten zusammen, um ihre gegenseitige politische Solidarität zu stärken und weiterzuentwickeln.“

Das Solidaritätsprinzip verkörpere zusammengefasst die „raison d'être“ der Europäischen Union, den Ausgleich zwischen Markt und Sozialstaat sowie insgesamt den Zusammenhalt in der Union. Die Funktion des Solidaritätsprinzips im EU-Recht bleibe aber unklar.

\section{Die Haushaltsdisziplin der US-Bundesstaaten als Vorbild für Deutschland und Europa}

Christian Deubner referierte zum Spannungsfeld von Solidarität versus ,moral hazard ' bei Staatsschuldenkrisen. ,Moral hazard' bezeichne risikoreiches Verhalten beziehungsweise die Unterlassung risikoaversen Verhaltens als Folge einer bestehenden Absicherung gegen die Folgen dieses Verhaltens. In der Euroschuldenkrise betrieben die überschuldeten Staaten ,moral hazard': In Erwartung eines Bailouts dämpften sie ihre Bemühungen zur Sanierung der Haushalte, da der Staatsbankrott eines oder mehrerer Eurostaaten unabsehbare Konsequenzen für die gesamte Eurozone nach sich ziehe, sodass die Gemeinschaft gezwungen sei, für die Folgen des Verhaltens der überschuldeten Staaten aufzukommen. Deubner verglich das Prinzip der Solidarität in den beiden Bundesstaaten Deutschland und USA. Beide seien Prototypen unterschiedlich strukturierter föderaler Systeme: kooperativ (Deutschland) und kompetitiv (USA).

Der entscheidende fiskalpolitische Unterschied zwischen beiden Bundesstaaten bestehe darin, dass der Bund in Deutschland die Schulden der Bundesländer garantiert und dies in den USA nicht der Fall ist.

In den USA sei aus diesem Grund der Schuldenstand der Gliedstaaten geringer und liege zwischen 8 und 27 Prozent des Bruttoinlandsprodukts (BIP). Dafür variierten die von den Gliedstaaten zu zahlenden Zinssätze für Kredite entsprechend der jeweiligen Verschuldung stärker als dies in Deutschland der Fall sei, da eine Garantie der Schulden durch den Bund fehle. In Deutschland zahlten alle Bundesländer hingegen einen ähnlich niedrigen Zinssatz. Die Garantie des Bundes für die deutschen Länder biete diesen keinen Anreiz für eine verantwortungsvolle Haushaltspolitik. In der Bundesrepublik betrage der Schuldenstand der Bundesländer zwischen 7 und 70 Prozent ihres jeweiligen BIP.

Was folgt daraus für die Europäische Union und die gegenwärtige Schuldenkrise? Insgesamt zeige sich, dass die Bundesstaaten, die eine kompetitive Struktur aufweisen, eine stärkere Haushaltsdisziplin einhalten. In der Analyse der Europäischen Union falle auf, dass sie bis zum Jahr 2009 aufgrund der No-BailoutKlausel ähnlich wie die USA strukturiert gewesen sei, was den Wettbewerb zwischen den Gliedstaaten betreffe. Allerdings habe sich in der Staatsschuldenkrise die Europäische Union seit 2009 stärker an dem kooperativen Modell Deutschlands orientiert, da die NoBailout-Klausel nicht mehr beachtet und mas- 
sive Finanzhilfen an einzelne Mitgliedstaaten gezahlt werden. ${ }^{1}$

Ulrich Brasche argumentierte in der anschließenden Diskussion, dass diese Überlegungen zu spät kämen. Die Staaten, die einen Bailout bräuchten, könnten die anderen solventen Staaten mit dem Argument erpressen, sie würden diese Staaten mit in den Abgrund reißen. Es fehle ein Mechanismus, Staaten und Banken geordnet insolvent gehen zu lassen. Deubner sagte, dass man zwei Strategien brauche: eine Strategie, die für den Normalfall der fiskalischen Politik gelte, und eine, die dazu diene, mit dem riesigen Schuldenberg in Europa umzugehen. Diese Politiken sollten nicht identisch sein. So sei der Vorschlag des deutschen Sachverständigenrates aus seinem Jahresgutachten 2011 zur Einführung eines Schuldentilgungsfonds sinnvoll. ${ }^{2}$ Der Vorschlag sehe vor, dass ein Schuldentilgungsfonds die Schulden der Mitgliedstaaten, deren Staatsverschuldung mehr als 60 Prozent ihres BIP entspreche, übernimmt, in einem gemeinsamen Fonds zusammenfasst und sie dann gemeinsam von allen Mitgliedstaaten der Europäischen Union getilgt werden. Dadurch sänken die Zinssätze, die überschuldete Staaten für ihre Kredite zahlen müssen, und die Schulden würden stärker beherrschbar. Anschließend könne man leichter Regeln durchsetzen, die, wie beim Fiskalpakt, bei einer normalen Verschuldung bis etwa 60 Prozent des BIP anwendbar sind.

\section{Die schwierige Suche nach einer neuen Rechtsordnung der Wirtschafts- und Währungsunion}

Peter-Christian Müller-Graff referierte über die rechtliche Neuordnung der europäischen Wirtschafts- und Währungsunion. Ihr Ziel bestehe in der Sicherung der Preisstabilität. Aufgrund ihres nationenübergreifenden und rechtlich unvollendeten Charakters stelle dies eine große Herausforderung dar.
Ein schwerwiegendes Problem habe in der anfänglich fehlenden Möglichkeit der Sanktionierung von Haushaltsdefiziten von Mitgliedstaaten der Eurozone bestanden. Ein weiteres Defizit stelle die sogenannte No-Bailout-Klausel des Art. 125 AEUV dar. Er verbiete die Haftung von Mitgliedstaaten für Verbindlichkeiten anderer Staaten. Allerdings untersage die Klausel keine Selbsthilfe. Das bedeute, die Staaten der Eurozone hätten das Recht, den Fortbestand der gemeinsamen Währung durch gegenseitige Hilfeleistungen zu gewährleisten und damit schädliche Folgen für ihre eigenen Volkswirtschaften abzuwenden. Für diese Lesart des Art. 125 AEUV spreche, dass Normen nicht widersinnig interpretiert werden dürften. Die gegenseitige Hilfe müsse aber auf freiwilliger Basis erfolgen, es gebe kein rechtliches Gebot der Unterstützung für notleidende Staaten.

Die seit Mai 2010 getroffenen Maßnahmen zur rechtlichen Neuordnung und finanziellen Rettung der Wirtschafts- und Währungsunion stellten einen Aufbruch in ein neues Territorium dar, die Union sei auf der Suche nach einer bislang nicht gefundenen befriedigenden Gesamtform für die Eurozone. Ein Bündel verschiedener Maßnahmen sei verabschiedet worden, welches unterschiedliche juristische Teilbereiche umfasse: Unionsrecht, transnationales Recht außerhalb des Primärrechts, privates Recht und nationales Recht. Bei den getroffenen Maßnahmen zeige sich der sukzessive Charakter der Lösung der Eurokrise und der Beseitigung ihrer Ursachen. Eine Änderung der Verfasstheit der Wirtschafts- und Währungsunion scheitere bislang an Großbritannien. Aus diesem Grund wurden der Europäische Stabilitätsmechanismus (ESM) und der Fiskalpakt als internationale Rechtsgebilde außerhalb des Unionsrechts entworfen und verabschiedet. Der ESM weise den Charakter einer eigenständigen internationalen Finanzinstitution auf, die den Eurostaaten mit bis zu 700

1 Siehe dazu auch Christian Deubner: Lehren für die Eurozone aus dem bundesdeutschen und US-amerikanischen Föderalismus, in diesem Band, S. 48-60.

2 Sachverständigenrat zur Begutachtung der gesamtwirtschaftlichen Entwicklung: Verantwortung für Europa wahrnehmen. Jahresgutachten 2011/12, Wiesbaden, November 2011. 
Milliarden Euro Kapital zu Hilfe kommen könne. Ziel des Fiskalpakts sei es, die Haushaltsdisziplin in den Eurostaaten und den anderen teilnehmenden EU-Staaten zu stärken und künftigen Schuldenkrisen vorzubeugen.

Für die weitere Ausgestaltung der Wirtschaftsund Währungsunion solle möglichst das Primärrecht genutzt werden. Aufgrund der britischen Blockadehaltung bliebe jedoch nur das internationale Recht außerhalb des Unionsrechts und vermutlich müsse eine Neuordnung der Wirtschafts- und Währungsunion weiter über diesen Weg beziehungsweise eine Kombination aus Unionsrecht und transnationalem Sonderrecht erfolgen.

\section{Auf dem Weg zu einer Theorie der Desintegration}

Annegret Eppler und Henrik Scheller konzeptionalisierten in ihrem Vortrag Desintegration und Integration theoretisch. Ausgangspunkt ihrer Überlegungen war die Finanz- und Wirtschaftskrise, die zu einer Zunahme desintegrierender Faktoren in Europa führe. Die bisherigen Theorien in der europäischen Integrationsforschung hätten einen ,normative bias ${ }^{\text {" } 3}$ und berücksichtigten desintegrative Tendenzen nicht ausreichend. Eppler und Scheller stellten die These auf, dass Desintegration ein Bestandteil des europäischen Integrationsprozesses sei und jede Zugkraft im Sinne von Integration eine desintegrative Gegenkraft in sich berge.

„integratio“ bedeute die (Wieder-)Herstellung einer Einheit (aus Differenziertem). Es gehe um die Verbindung einer Vielheit von Personen oder Gruppen zu einem größeren Ganzen. Desintegration meine hingegen die Auflösung beziehungsweise Spaltung eines Ganzen in seine Teile. Integration oder Desintegration sollte man eher als graduelle Konzepte verstehen und nicht als einen feststehenden Zustand. Wesentlich sei die Gleichzeitigkeit der Prozesse von
Integration und Desintegration. Formen von Integration stellten Vertragsänderungen, Vertiefungen oder Erweiterungen dar.

Für die theoretische Untermauerung von Integration und Desintegration sei zuerst festzuhalten, dass die tradierten Integrationstheorien kombiniert und weiterentwickelt werden müssten, um Desintegration theoretisch gut erfassen zu können. Föderalismustheorien würden sich als Basis für eine theoretische Erfassung von Integration und Desintegration besser als Integrationstheorien eignen, da sie Prozesse von Zentralisierung und Dezentralisierung nicht bewerten.

Die abhängige Variable einer Theorie von Desintegration bestimme sich durch das Integrationsniveau in der Europäischen Union. Die Messung des Integrations- oder Desintegrationsniveaus ließe sich absolut oder relational bestimmen. Eine relationale Messung könne zum Beispiel die Finalitätsvorstellungen in der Union als Referenzwert nutzen.

Die unabhängige Variable stellten Faktoren dar, die eine Verschiebung des Integrationsniveaus zur Folge hätten. Kräfte mutierten erst zu Zug- und Gegenkräften der Integration durch intervenierende Variablen. Intervenierende Variablen seien unter anderem Zeit, Krisensituationen, Akteurskonstellationen und die Präferenzen der Akteure. Beispiele für Gegenkräfte fänden sich in der sozioökonomischen oder kulturellen Diversität der Mitgliedstaaten, die Spannungen im Integrationsprozess hervorrufen könnten.

Insgesamt müsse Integration als ein dialektischer und dynamischer Prozess verstanden werden. Eine kontrollierte Desintegration könne dabei auch zu einer Stärkung von Integration führen. Weiterhin müssten Impulse der Integration und der Desintegration von außerhalb des Mehrebenensystems erfasst und akteurs- und strukturzentrierte Ansätze für die Analyse kombiniert werden.

3 Anne Faber/Wolfgang Wessels: Die Verfassungskrise der EU als Krise „der“ Integrationstheorie? Plädoyer für eine Neufokussierung der theoretischen Debatte; in: Zeitschrift für Internationale Beziehungen 2/2005, S. 353-359. 
Europäische Solidarität am Beispiel der Energiepolitik

Nadine Piefer trug zum Thema ,Solidarität in der Energiepolitik" vor und definierte zuerst den vielseitigen Begriff der Solidarität. So umfasse Solidarität eine wechselseitige Verpflichtung, ein „Wir-Gefühl“, sei reziprok, stelle ein Prinzip der gegenseitigen Rückversicherung dar und könne auch als wohlverstandener Eigennutz betrachtet werden, der durch Einheit Stärke erzeuge. Das Prinzip der Musketiere, „Einer für alle, alle für einen“, diente als Beispiel. Solidarität verfüge über eine horizontale Dimension, die gegenseitige Solidarität auf gleichberechtigter Basis, und über eine vertikale Dimension, bei der Wohlhabende Ärmeren helfen und die Beziehung hierarchisch strukturiert sei. Weiterhin könne Solidarität zwischen verschiedenen Staaten (internationale Solidarität) aber auch zwischen unterschiedlichen Gesellschaften (transnationale Solidarität) bestehen.

Bezogen auf die Energiepolitik werde Solidarität in Art. 194 AEUV erwähnt: „Die Energiepolitik der Union verfolgt im Geiste der Solidarität zwischen den Mitgliedstaaten im Rahmen der Verwirklichung oder des Funktionierens des Binnenmarkts und unter Berücksichtigung der Notwendigkeit der Erhaltung und Verbesserung der Umwelt folgende Ziele“.

Als Ziele der europäischen Energiepolitik werden im Wesentlichen die Sicherung des Energiemarktes, die Garantie des Wettbewerbs, die Versorgungssicherheit und die Energieeffizienz genannt. Das Prinzip der Solidarität zeige sich in erster Linie bei der Versorgungssicherheit. Die Spezifizierung, wie im Krisenfall die Versorgungssicherheit gewährleistet werden könne, sei aber noch nicht erfolgt. So bestehe zum Beispiel beim Erdöl die Verpflichtung der Mitgliedstaaten eigene Reserven anzulegen, aber es fehle ein Mechanismus, der eine solidarische Verteilung des Erdöls unter den EUStaaten im Krisenfall regelt.
Die internationale Energiesolidarität, das heißt die Energiepolitik der Europäischen Union, die über ihre eigenen Grenzen hinausgehe, verfüge über zwei Dimensionen: Zum einen bemühe sich die Europäische Union um eine Diversifikation der Lieferanten sowie um gute Beziehungen zu selbigen und zum anderen nehme der globale Wettbewerb im Energiebereich zu. Die Europäische Union betreibe als zentrales Instrument der Energiepolitik Energiedialoge mit strategischen Partnern und wichtigen Lieferanten. Die wachsende Nachfrage nach Energie werde eine stärkere internationale Solidarität erschweren, insbesondere China, Indien und andere aufstrebende Staaten würden künftig mehr Energie benötigen.

\section{Ein verstärkter Intergouvernementalismus in der Finanz- und Wirtschaftspolitik}

Franz Cromme referierte über den ESM und den Fiskalpakt als mögliche Fortentwicklungen der Europäischen Union als Staatenverbund. Es stellte sich für Cromme vor allem die Frage, wie sich die Europäische Zentralbank (EZB) als unabhängige Institution in dieses neue System einfügen werde. Die EZB sei bislang unabhängig und auf das Ziel der Preisstabilität festgelegt. Sie solle aber auch die nationalen Wirtschaftspolitiken unterstützen, solange das Ziel der Preisstabilität beachtet werde. Die EZB dürfe nicht auf eine kapitalmarktunabhängige Finanzierung der Staatsschulden hinarbeiten, da diese Politik sonst ihre Unabhängigkeit gefährde. Die Garantie der Unabhängigkeit der EZB zur Sicherung der Preisstabilität sei entscheidend. Eine (Selbst-) Beschränkung der Rolle der EZB wäre einfacher, wenn der ESM auch Banken direkt finanzieren könnte. Mit dem Aufbau einer europäischen Bankenaufsicht werde die kapitalmarktunabhängige Finanzierung der Staatsschulden durch ESM und Fiskalpakt an Bedeutung verlieren. Der Kapitalmarkt müsse künftig wieder die zentrale Rolle bei der Finanzierung der Staatsschulden spielen. 
Für die Finanz- und Wirtschaftspolitik der Europäischen Union könne in Zukunft ein Entscheidungsverfahren genutzt werden, das bereits in der Gemeinsamen Außen- und Sicherheitspolitik (GASP) Anwendung findet. Der Ministerrat würde so zu einer Art „Wirtschaftsregierung". Die Europäische Kommission solle ebenfalls eine wichtige Rolle spielen. So könnte das Amt eines ,europäischen Finanzministers", ähnlich dem Amt der Hohen Vertreterin der Union für Außen- und Sicherheitspolitik, geschaffen werden.

Insgesamt zeigte die Tagung, dass die Desintegration sowohl Chancen als auch Risiken für die künftige Gestaltung des europäischen Projekts bietet. Begriff und Verständnis von Desintegration sind bislang zu wenig erforscht und die Beschäftigung mit der Europäischen Union konzentriert sich zu stark auf immer weitere Schritte der Vertiefung und Erweiterung.

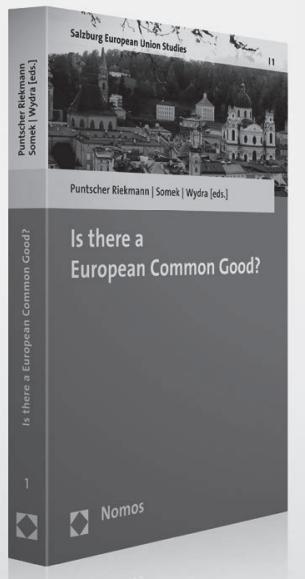

Diese Studie spiegelt aus unterschiedlichen Perspektiven die Herausforder-

ungen rund um die Definition des europäischen Gemeinwohls wider.
Solidarität hingegen ist ein integraler Wert jeder Union und beinhaltet die gegenseitige Treue und Zuverlässigkeit in einer Gemeinschaft. In einer Krise zeigen sich Ausmaß und Grenzen der gegenseitigen Solidarität. Der Wert der Solidarität muss sich dabei auf ein solides Fundament gründen, um eine starke Gemeinschaft zu bilden. Die Tagung hat gezeigt, dass bereits viele Maßnahmen getroffen wurden, um die wirtschaftliche und finanzielle Solidität in der Europäischen Union voranzutreiben. Dieser Aufbau eines wirtschaftlich und politisch stabilen Fundaments in Europa muss weiter vorangetrieben werden, um langfristig die solidarische Union der europäischen Staaten zu erhalten und fortzuentwickeln. $\mathrm{Ob}$ sich alle Mitgliedstaaten auf eine gemeinsame Vision einer soliden und solidarischen Union einigen können und ob der Austritt einiger Staaten den Aufbau eines stärkeren Europas befördern würde, sind offene Fragen.

\section{Is there a European Common Good?}

Herausgegeben von Sonja

Puntscher Riekmann, Alexander

Somek und Doris Wydra

2013, 275 S., brosch., 44,- $€$

ISBN 978-3-8329-7976-8

(Salzburg European Union Studies, Bd. 1)
Weitere Informationen: www.nomos-shop.de/19956 